\title{
Effects of Health Information Access and Health Service Access on Health Literacy and Health Behavior
}

\author{
Niken Lastiti Veri Anggaini*, Bambang Supriyono, Lely Indah Mindarti, Firda Hidayati \\ Department of Public Administration, Faculty of Administrative Science \\ Universitas Brawijaya \\ Malang, Indonesia \\ *nik_fia@yahoo.co.id
}

\begin{abstract}
Health is one of the most important factors in the development of a country. Good health will affect one's productivity. The better the health status of a country is expected to also be able to increase the degree of development in other sectors. A person's health status is greatly influenced by health behavior. This study tries to explore the factors that influence health behavior based on the variables of access to health information, access to health services and use of health literacy as mediation. This type of research is included in explanatory research with a quantitative approach. The data analysis method used was SEM GesCA. The results showed that there was a significant effect between access to health information and health literacy with the Path Coefficient value of 0.093. This differs in the effect of access to health information on health behavior which shows an insignificant effect with the Path Coefficient of 0.059. However, if mediated using health literacy, the result becomes a significant effect with a coefficient of 0.0298 . Meanwhile, the health service access variable has a significant effect on health literacy with a Path Coefficient of 0.094. Likewise, the effect of access to health services on health behavior, which showed a Path Coefficient of 0.149 which means a significant effect.
\end{abstract}

Keywords-health information access, health service access, health literacy, health behavior

\section{INTRODUCTION}

The practice of administering health services in Indonesia so far still raises many problems, such as institutional problems, service management and professionalism of government officials. The problems faced by the Indonesian bureaucracy do not only come from the internal bureaucracy, but also come from externals, namely the dynamics of society including the development of problems faced by the community. Several health service problems in Indonesia cover accessibility, capability, capacity and affordability problems. First is related to availability of good health facilities that are concentrated in big cities. The second related to number of specialist doctors which is still a little compared to general doctors. The third is related to the availability of medical devices with innovative breakthroughs is limitedAnd the last is related to the affordability of society to health costs.

The health sector is a part that is closely related to public services. Increasing public demands for public services, especially health, this is related to the growing needs of the community and advances in information technology. In general, everyone agrees that health is an important thing in life. Health degree will affect a person's performance and productivity. A person's health status is determined by their health behavior, namely how an individual makes decisions related to their health.

Health is one of the dominant factors in maintaining the sustainability of human life and the environment. However, in fact, there are still many behaviors that do not reflect clean and healthy living habits. This then becomes the background for the emergence of various behavioral studies (including in the health sector) to find the factors that influence the formation of individual, group and organizational behavior. This study tries to explore the factors that influence health behavior based on variables of health information access, health services access, and health literacy.

In implementing a health service system, access to health services and access to information is the core. Health service itself is a form of effort organized by an organization that aims to maintain, improve health, prevent and cure diseases and restore public health by meeting the needs of patients by providing effective and efficient services. Gulliford et al. [1] stated that health services accessibility can be measured build upon the service, reach ability and physical accessibility. The better the access to health services that is received by the community, it is hoped that the community's involvement in health programs will also increase which in turn will encourage the achievement of improvements and increase in the degree of public health through clean living habits.

Apart from access to health services, another factor that also affects a person's health grade is access to health information. Information is one of the human needs that cannot 
be negated in giving effect to a person's health behavior. According to Fiorino [2], information is a basic and important element in a participatory process to encourage informed decision making. In other word, Reed [3] said that "The need for scientific information and analysis to inform stakeholder deliberation has been identified by many authors as an essential ingredient in any participatory process. "

The importance of access to information was also conveyed by the International Association for Public Participation, which argues that the need of information from entrants is essence for pre-eminent participatory processes in every society: Public participation have to supply information needed by participants in order to participate in a valuable means" [4]. In a wider scene, sufficient information access is the most important thing for a democratic society, and the inadequacy of information will hinder political converse and democratic discourse [5]. Therefore, access to appropriate information supplies is one of the main indicators in participatory process assessment [6-8].

The third variable is the variable for the level of health literacy. The role of health literacy in health behavior and increasing community involvement in health programs cannot be ignored. Literacy is an important ability for someone to have because even by having the most basic literacy skills, a person can further increase their knowledge and potential to achieve their goals so that they can participate more in society, both economically and socially. For an individual, health literacy is determined by the level of education, culture, and language. However, besides that, communication skills and assessing interactions with other people are also needed related to health as well as the ability of the media, market and government to provide health information correctly [9].

The novelty in this research is there is no research before that link the three variables (health information access, access to health services, health literacy) in shaping health behavior, especially intend to explore effect of health information access and health service access on health behavior and use of health literacy as mediation variable.

\section{MATERIALS AND METHODS}

This study used explanatory research with quantitative approach. The data collection techniques used a questionnaire that was distributed to 278 respondents aged $\geq 18$ years. This study took the research location in Blitar City which includes three districts, namely Sananwetan, Kepanjenkidul and Sukorejo districts. This is based on the consideration of the results of the Public Health Development Index (IPKM) in 2018 which placed Blitar City in first place where the result IPKM 2013, Blitar City was still in the 8th position. Data analysis used SEM GesCA. This study aims to determine the effect of health services and health information access on health behavior. This study also tries to explore the use of health literacy as a mediating variable that connects access to health information and health behavior as well as access to health services and health behavior.

\section{RESULTS}

\section{A. Measurement Model}

Based on the data in Table 1, it can be explained that all indicators are significantly able to explain the variables. The measurement model for the Information Access Variable also informs that the Modern Equipment Indicator (X1.3 has the greatest loading factor value, which is $79.8 \%$ with a mean value of 3.89. This means that Modern Equipment (X1.3) is the most dominant indicator in measuring the Access to Information Variable. The Health Service Access Variable Measurement Model also informs that the Barriers Indicator (X2.3) and the availability indicator (X2.1) have almost the same loading factor values, namely 0.835 and 0.834 . The Health literacy Variable Measurement Model also informs that the Communicative Health Literacy Indicator (Y2.2) has the biggest loading factor value.

TABLE I. Results of the Descriptive ANALysis AND the MEASUREMENT Model of EACH VARIABLE

\begin{tabular}{|c|c|c|c|c|c|}
\hline Variable & Indicator & Loading Factor & Mean & SE & $\mathbf{C R}$ \\
\hline \multirow{4}{*}{ Health Information Access (X1) } & X1.1 Easy of nafigation & 0.748 & 3,62 & 0.059 & $12.71 *$ \\
\hline & X1.2 Convenience & 0.665 & 3,58 & 0.057 & $11.58^{*}$ \\
\hline & X1.3 Modern Equipment & 0.798 & 3,89 & 0.015 & $53.34 *$ \\
\hline & X1.4 Self Relience & 0.782 & 3,76 & 0.009 & $86.01 *$ \\
\hline \multirow{3}{*}{ Health Service Access (X2) } & X2.1 Availability & 0.834 & 3,67 & 0.021 & $39.74 *$ \\
\hline & X2.2 Insurance & 0.737 & 3,67 & 0.023 & $32.01 *$ \\
\hline & X2.3 Resistance & 0.835 & 3,73 & 0.018 & $47.21 *$ \\
\hline \multirow{3}{*}{ Health Literacy (HL) (X1) } & X1.1 Functional HL & 0.611 & 3.31 & 0.040 & $15.45^{*}$ \\
\hline & X1.2 Communicative HL & 0.867 & 3.89 & 0.012 & $73.85^{*}$ \\
\hline & X1.3 Critical HL & 0.844 & 4.03 & 0.009 & $98.93 *$ \\
\hline \multirow{3}{*}{ Health Behavior (Y1) } & Y.1.1 Health maintenance & 0.870 & 4,10 & 0.014 & $64.36^{*}$ \\
\hline & Y.1.2 Health seeking behavior & 0.798 & 3.97 & 0.012 & $64.34 *$ \\
\hline & Y.1.3 Environmental health behavior & 0.819 & 4.04 & 0.022 & $37.31 *$ \\
\hline
\end{tabular}

$\mathrm{CR}^{*}=$ significant at .05 level 
It showed that Communicative Health Literacy (Y2.2) is the most influential indicator in Health Literacy mensuration. Variable with a loading factor value of 0.867 and a value The mean is 0.867. And for the measurement model, the Health Behavior variable is also informing that the health maintenance indicator (Y3.1) has the greatest loading factor value, which is equal to 0.870 with a mean value of 4.10 . This means that health maintenance (Y3.1) is the most influential indicator in determine the Health Behavior.

\section{B. Analysis results: Structural Equation Modeling (SEM) GesCA}

1) Goodness of Fit Model: In general, in order to notice whether the model established in the research is good or not, it can be measured from the goodness of fit of the structural model and the goodness of fit of the overall model. The results of testing the goodness of fit of the structural model and the overall model in accordance with the results of the GesCA analysis are presented in the Table 2 below.

TABLE II. THE GOODNESS OF FIT MODEL TEST

\begin{tabular}{|c|c|}
\hline \multicolumn{2}{|c|}{ Model Fit } \\
\hline FIT & 0.492 \\
\hline AFIT & 0.488 \\
\hline GFI & 0.989 \\
\hline
\end{tabular}

Based on Table 2, GFI has met the cut off value $\geq 0.90$. It means that the GesCA model is appropriate for use in the study. This is based on the parsimony principle (the principle of simplicity), if one of the Goodness of Fit Overall Models has met the cut off, the model is said to be feasible and good.

FIT and AFIT were used to size Goodness of Fit Structural models. In this study, the FIT value is 0.492 . It can be said that the model can define all the present variables by $49.2 \%$. Diversity of Health Information Access (X1), Health Services Access (X2), Health Literacy (Y1), and Health Behavior (Y2) that can be described by the model is $49.2 \%$ and the residual $(50.8 \%)$ could be clarified by diverse variables which were not involved in the research. It can be concluded that the model developed in the study is good.
Adjusted FIT (AFIT) is almost the same as FIT. The values of AFIT is 0.488. It means that the model established can illustrate all variables by $48.8 \%$. The diversity of Information Access (X1), Access to Health Services (X2), Health Literacy (Y1), and Health Behavior (Y2) that can be verified by the model is $48.8 \%$ and the residual $(51.2 \%)$ could be defined by diverse variables. Finally, it can be concluded that the model established is good.

\section{Hypothesis Test}

TABLE III. HYPOTHESIS TEST

\begin{tabular}{|l|l|c|c|c|}
\hline \multicolumn{1}{|c|}{ Exogenous } & Endogenous & Path Coefficient & SE & CR \\
\hline Information access & Health Literacy & 0.093 & 0.044 & $2.11 *$ \\
\hline Health Service Access & Health Literacy & 0.094 & 0.046 & $2.02 *$ \\
\hline Health literacy & Health Behavior & 0.321 & 0.071 & $4.51 *$ \\
\hline Information Access & Health Behavior & -0.059 & 0.057 & 1.04 \\
\hline Health Service Access & Health Behavior & 0.149 & 0.063 & $2.38^{*}$ \\
\hline
\end{tabular}

$\mathrm{CR}^{*}=$ significant at .05 level

Based on Table 3, the results of the analysis showed that almost all the relationships between variables on the direct effect show significant results, but there is one direct effect that is not significant, namely the relationship between Health Information Access (X1) and Health Behavior (Y2). This means that Access to Health Information (X1) has no significant effect on Health Behavior (Y3). In table 3 above, it can also be seen that the dominant influence on health behavior is health literacy with a Path Coefficient value of 0.321 . Meanwhile, the effect of health information and health services access on health literacy resulted in almost the same values, namely 0.093 and 0.094

\section{Mediation Testing}

Mediation testing is intended to see the indirect effect between two variables. In this research, health literacy variable is used as a mediating variable that connects health information access and health behavior and between health services access and health behavior. More details can be seen in table 4 below:

TABLE IV. RESEARCH HYPOTHESIS TESTING (INDIRECT EFFECT)

\begin{tabular}{|l|l|l|l|}
\hline \multicolumn{1}{|c|}{ Correlation } & Coefficient & Description \\
\hline $\begin{array}{l}\text { Health Information Acces (X1) -> Health } \\
\text { Literacy (Y1) -> Health Behavior (Y2) }\end{array}$ & 0,0298 & $\begin{array}{l}\text { Health Information Acces (X1) -> Health Literacy (Y1) (Sig.), } \\
\text { Health Literacy (Y1) -> Health Behavior (Y2) (Sig.) }\end{array}$ \\
\hline $\begin{array}{l}\text { Health Service Access (X2) -> Health } \\
\text { Literacy (Y1) -> Health Behavior (Y2) }\end{array}$ & $\begin{array}{l}\text { Health Service Access (X2) -> Health Literacy (Y1) (Sig.), } \\
\text { Health Literacy (Y1) -> Health Behavior (Y2) (Sig.) } \\
(0,0302<0,149) \text { where 0,149 is coefficient of direct effect X2 on Y2 }\end{array}$ & $\begin{array}{l}\text { Not Significant } \\
0,0302\end{array}$ \\
\hline
\end{tabular}

Based on table 4 above, the indirect effect between access to health information on health behavior through health literacy results in a Path Coefficient of 0.0298. This shows that the higher the Access to Health Information (X1), the higher the
Health Behavior (Y2) through the improvement of the Health Literacy (Y1) pathway. The variable Health Literacy (Y1) is a perfect mediation variable in the relationship between Access to Health Information (X1) and Health Behavior (Y2), because 
the direct effect of Health Information Access (X1) on Health Behavior (Y2) has no significant effect.

This is different from the results of testing the effect of Health Service Access (X2) on Health Behavior (Y2) through Health Literacy (Y1) which showed no take effect significantly by a path coefficient of 0.0302 . Meanwhile, the direct influence that consist of the effect of health service access (X2) on Health behavior (Y2) and health literacy (Y1) on health behavior (Y2) are significant. The coefficient of indirect influence is smaller than the coefficient of direct influence between Health Service Access (X2) and Health Behavior (Y2) or $0.0302<0.149$. This showed that there is no indirect influence in the link between Health Service Access (X2) and Health Behavior (Y2) through Health Literacy (Y1). The Health Literacy variable (Y1) is not a mediating variable on the relationship between Health Service Access (X2) and Health Behavior (Y2).

\section{E. Discussion}

Several theoretical concepts regarding health literacy have been developed, one of which is by Sørensen, et al. [10]. They said that literacy concept has an important component in health literacy because in this concept there are three parts of health. The three components are health care capability, disease prevention efforts and health education or promotion which will generate knowledge and skills in health care. The factors that cause the level of health literacy to be inadequate are influenced by the level of education, economy or household income, cultural culture, exercise, age, physical examination, smoking habits and access to health information [11]. Access to health information is the most influential factor in a person's health literacy [12]. To make decisions in terms of promoting good health requires extensive health information, easily accessible according to the necessary needs and conditions of the individual cultural environment

The research showed that health information access has a notable influence on health literacy. Access is an opportunity to obtain or use certain resources. It is in line with research conducted by Ishikawa et al. [12], access to health information is the most influential factor in a person's health literacy. The results also reinforced the study held by Sun et al. [13], access to health information can affect a person's health literacy, besides education, age, socio-economic, and occupational factors. According to Bohlman et al, health literacy is determined by the level of education, culture, and language. In addition, skills in communicating and interacting with others related to health and the ability of the media, the market and the government are also needed to provide health information correctly [9].

Measuring health literacy in this study used a method developed by Ishikawa et al. [12] and colleagues known as the health literacy scale (HLS-14). There are 14 question items consisting of functional Health Literacy (five items), communicative Health Literacy (five items), and critical Health Literacy (five items). Functional health literacy is related to elementary skills in reading and writing health information.
Communicative health literacy is a person's ability to extract information and obtain meaning from various forms of communication carried out related to his health. Meanwhile, critical literacy is related to a person's ability to evaluate, critically analyse a variety of existing health information and use it in making health-related decision

So far, research related to the effect of health information access on health behavior is more focused on the use of information media such as research conducted by EscobarChaves et al. [14], where the mass media has been shown to influence various behaviors related to adolescent health. Another study showed that media has a relationship with adolescent sexual behavior which is considered to be as important as family, school, and peers. Furthermore, it was found that health information access had an effect on health behavior even though the effect was not significant. However, different results are shown when the variables access to health information and health behavior are mediated by the health literacy variable, which has a significant effect. This shows that the large number of access to information does not necessarily affect a person's health behavior if it is not supported by good health literacy. Health literacy is a key factor in the relationship between access to health information and an individual's health behavior.

In addition, the results of the study indicate a significant influence between access to health services and health literacy. Pawlak [15] proposes a determinant of health literacy model, where the determinants that affect a person's health literacy include age, genetics, language, race and ethnicity, education, occupation, socio-economic status and environmental factors. Forsyth et al. [16] argue that access to health services depends on the location of the service, the means of transportation available to reach health services, and the existence of health insurance / insurance. Access to health services will also affect a person's ability to obtain health information. Research on health literacy conducted in USA 2003 (National Assessment of Adult Literacy), showed that there is a relationship between one part of access to health services, namely health insurance with the level of health literacy. As many as 53\% of the population without health insurance has a low level of health literacy [17]. The study of Bains and Egede [18] also showed a similar thing, namely that people who do not have health insurance have a much higher proportion of low health literacy rates.

It also supported research by Kohan et al. [19]. They stated that the success of health services is not only determined by the quality of the program and the health workers involved, but how the patient's understanding of accessing and using these services also plays a role in the success of health services. According to Gulliford et al. [1], health service measurement can be conducted based on result of the utilization that determined by several aspects such as affordability, physical accessibility, and the services received. Furthermore, for the effectiveness of health services, they also argued that the presence of service supplies and service barriers have to be considered in the distinct views. Finally, there are many aspects 
have to be considered in measuring quality of health service access. It is also necessary to pay attention to the needs and demands of health services, as well as the cultural aspects of society.

\section{CONCLUSION}

Health behavior reflects a person's attitude in making decisions related to his health. There are many factors that influence the formation of an individual's health behavior, both internal to the individual and the result of collaboration with the surrounding environment. This study found that health services access and health literacy had a prominent influence on health behavior. Meanwhile, information access has no significant effect on health behavior, yet these results are different when mediated by health literacy that produce a significant effect. This shows that health literacy is a perfect mediation between access to health information and health behavior. Meanwhile health literacy do not mediation variable in the relation between health service access and health behavior.

\section{CONFLICTS OF INTEREST}

The authors affirm no conflict of interest in this study.

\section{REFERENCES}

[1] M. Gulliford, J. Figueroa-Munoz, M. Morgan, D. Hughes, B. Gibson, R. Beech, and M. Hudson, "What Does' Access to Health Care'mean?," J. Health Serv. Res. Policy, vol. 7, no. 3, pp. 186-188, 2002.

[2] D.J. Fiorino, "Citizen Participation and Environmental Risk: A Survey of Institutional Mechanisms," Sci. Technol. Hum. Values, vol. 15, no. 2, pp. 226-243, 1990.

[3] M.S. Reed, "Stakeholder Participation for Environmental Management: A Literature Review," Biol. Conserv., vol. 141, no. 10, pp. 2417-2431, 2008

[4] International Association of Public Participation, "IAP2 Spectrum of Public Participation.," 2007.

[5] P.T. Jaeger, "Information Policy, Information Access, and Democratic Participation: The National and International Implications of the Bush
Administration's Information Politics," Gov. Inf. Q., vol. 24, no. 4, pp. 840-859, 2007

[6] P.B. Edwards, R. Hindmarsh, H. Mercer, M. Bond, and A. Rowland, "A Three-Stage Evaluation of a Deliberative Event on Climate Change and Transforming Energy,” J. public Delib., vol. 4, no. 1, 2008.

[7] G. Rowe and L.J. Frewer, "Public Participation Methods: A Framework for Evaluation," Sci. Technol. Hum. values, vol. 25, no. 1, pp. 3-29, 2000 .

[8] S. Tuler and T. Webler, "Voices from the Forest: What Participants Expect of a Public Participation Process," Soc. Nat. Resour., vol. 12, no. 5, pp. 437-453, 1999.

[9] D.A. Kindig, A.M. Panzer, and L. Nielsen-Bohlman, "Health Literacy: A Prescription to End Confusion," 2004.

[10] K. Sørensen, S. Van den Broucke, J.M. Pelikan, J. Fullam, G. Doyle, Z Slonska, B. Kondilis, V. Stoffels, R.H. Osborne, and H. Brand, "Measuring Health Literacy in Populations: Illuminating the Design and Development Process of the European Health Literacy Survey Questionnaire (HLS-EU-Q)," BMC Public Health, vol. 13, no. 1, pp. 1$10,2013$.

[11] Y.-B. Liu, L. Liu, Y.-F. Li, and Y.-L. Chen, "Relationship between Health Literacy, Health-Related Behaviors and Health Status: A Survey of Elderly Chinese,” Int. J. Environ. Res. Public Health, vol. 12, no. 8, pp. 9714-9725, 2015.

[12] H. Ishikawa and T. Kiuchi, "Health Literacy and Health Communication," Biopsychosoc. Med., vol. 4, no. 1, pp. 1-5, 2010

[13] Y. Sun, N. Wang, X. Guo, and Z. Peng, "Understanding the Acceptance of Mobile Health Services: A Comparison and Integration of Alternative Models," J. Electron. Commer. Res., vol. 14, no. 2, p. 183, 2013.

[14] S.L. Escobar-Chaves, S.R. Tortolero, C.M. Markham, B.J. Low, P. Eitel, and P. Thickstun, "Impact of the Media on Adolescent Sexual Attitudes and Behaviors," Pediatr. Ed., vol. 116, no. 1, pp. 303-326, 2005.

[15] R. Pawlak, "Economic Considerations of Health Literacy," Nurs. Econ., vol. 23, no. 4, p. 173, 2005.

[16] K. Forsyth and E. Hamilton, "Social Services Occupational Therapists' View of Practice and Integration with Health: A Survey," Br. J. Occup. Ther., vol. 71, no. 2, pp. 64-71, 2008.

[17] S. White, "Assessing the Nation's Health Literacy: Key Concepts and Findings of the National Assessment of Adult Literacy (NAAL): American Medical Association Foundation," Chicago, IL, 2008.

[18] S.S. Bains and L.E. Egede, "Association of Health Literacy with Complementary and Alternative Medicine Use: A Cross-Sectional Study in Adult Primary Care Patients," BMC Complement. Altern. Med., vol. 11, no. 1, pp. 1-8, 2011

[19] S. Kohan, S. Ghasemi, and M. Dodangeh, "Associations between Maternal Health Literacy and Prenatal Care and Pregnancy Outcome," Iran. J. Nurs. Midwifery Res., vol. 12, no. 4, 2008. 